\title{
A feedback loop enrchment analysis in gene network of bronchial asthma and pulmonary tuberculosis interaction
}

\author{
Evgeny S. Tiys \\ Laboratory of Computer-Assisted \\ Proteomics \\ Institute of Cytology and Genetics \\ SB RAS \\ Novosibirsk, Russia
}

\author{
Pavel S. Demenkov \\ Laboratory of Computer-Assisted \\ Proteomics \\ Institute of Cytology and Genetics \\ SB RAS \\ Novosibirsk, Russia \\ Nikolay A. Kolchanov \\ Systems Biology Department \\ Institute of Cytology and Genetics \\ SB RAS \\ Novosibirsk, Russia
}

\author{
Vladimir A. Ivanisenko \\ Laboratory of Computer-Assisted \\ Proteomics \\ Institute of Cytology and Genetics \\ SB RAS \\ Novosibirsk, Russia
}

\begin{abstract}
Feedback loops play a key role in management of processes including biological processes. In gene networks, feedback loops are presented by directed cycles, that can contain positive and negative regulatory links. We suggest that presence of such cycles can characterize gene networks build for biological processes. Earlier we developed a web-tool FunGeneNet, which can estimate enrichment of biologically meaningful networks with functional links. Based on this work, here we show that the network of interactions between bronchial asthma and pulmonary tuberculosis are enriched by short cycles.
\end{abstract}

Keywords - gene networks, random networks, enrichment analysis, feedback loops

\section{Introduction}

Feedback loops are known to play important regulatory role in biological processes [1-5]. It is know that feedback loops are significantly frequently occurs in biological networks in compare to random ones obtained by edge permutation [6]. Associative gene networks are useful tool for integration of various types of biological information. Previously, we showed that networks build for biological processes can be distinguished from random networks by number of edges [7]. The question arises as to whether the associative gene networks build for biologically meaningful sets of genes can be distinguished from the meaningless ones by the number of cycles in them? In this pilot work we show how to check it with a network of interaction between bronchial asthma and pulmonary tuberculosis [8] as an example.

\section{Materials and methods}

To build associative gene networks the ANDSystem database is used [9]. Random networks obtained as analyzed networks with swapped vertex labels were used to model biologically irrelevant networks. To faster the algorithm only vertices of the considered networks were swapped in compare to methods permuting the whole global network. A limitation of the swapping process is the following: swapped vertices have strictly the same degree in the global graph as it implemented in FunGeneNet [7]. A network of interactions between asthma and tuberculosis obtained earlier [8] and containing 19 genes was taken as a biologically meaningful network. Also we considered a subnetwork of this network, which is relevant to th1- and th2-immune responses including IL2, IL12, TNF, IFNG, IL1B, IL6, IL4, IL10 genes. We excluded undirected types of interactions from analysis before comparing the networks with random ones. The following types of interactions were considered: up- and down- regulation of expression, activity and degradation. Directed cycles of length 3 were counted in the analyzed and random networks.

\section{Results}

We obtained significant differences in the number of cycles for both whole network of bronchial asthma pulmonary pulmonary tuberculosis interaction and th1-th2network $(\mathrm{p}<0.001)$. These results support us to test if other biologically relevant sets of genes are enriched with the feedback loops. We plan to check whether the sets of genes associated with the Gene Ontology biological processes contain bigger number of cycles than randomly chosen sets of genes.

\section{ACKNOWLEDGMENT}

This work was supported by budget project AAAAA17-117092070032-4.

\section{REFERENCES}

[1] Jr. Ferrell, "Self-perpetuating states in signal transduction: positive feedback, double-negative feedback and bistability," Current opinion in cell biology, vol. 14, no. 2. pp. 140-148, 2002

[2] Yu Z et al. "A cyclin D1/microRNA 17/20 regulatory feedback loop in control of breast cancer cell proliferation," The Journal of cell biology, vol. 182, no. 3, pp. 509-517, 2008.

[3] S. Sun et al. "miR-146a and Krüppel-like factor 4 form a feedback loop to participate in vascular smooth muscle cell proliferation," EMBO reports, vol. 12, no. 1, pp. 56-62, 2011.

[4] J. Locke et al. "Experimental validation of a predicted feedback loop in the multi-oscillator clock of Arabidopsis thaliana," Molecular systems biology, vol. 2, no. 1, 2006.

[5] S. Brabletz et al. "The ZEB1/miR-200 feedback loop controls Notch signalling in cancer cells," EMBO reports, vol. 30, no. 4, pp. 770-782, 2011

[6] R. Milo et al. "Network motifs: simple building blocks of complex networks," Science, vol. 298, no. 5594, pp. 824-827, 2002.

[7] E. Tiys et al. "FunGeneNet: a web tool to estimate enrichment of functional interactions in experimental gene sets," BMC genomics, vol. 19, no. 3, pp. 76, 2018.

[8] E. Bragina et al. "Insights into pathophysiology of dystropy through the analysis of gene networks: an example of bronchial asthma and tuberculosis," Immunogenetics, vol. 66, no. 7-8, pp. $457-465,2014$.

[9] V. Ivanisenko et al. "ANDSystem: an Associative Network Discovery System for automated literature mining in the field of biology," BMC systems biology, vol. 9, no. S2, pp. S2, 2015. 